\title{
Polymer Matrix Nanocomposites and Nanostructured Materials
}

\author{
Luiz Antonio Ferreira Coelho, ${ }^{1}$ Sergio Henrique Pezzin, ${ }^{1}$ Marcio Rodrigo Loos, ${ }^{2}$ \\ Luis Antonio Sanchez de Almeida Prado, ${ }^{3}$ and Alejandro Manzano Ramirez ${ }^{4}$ \\ ${ }^{1}$ Center for Technological Sciences, State University of Santa Catarina, 89223-100 Joinville, SC, Brazil \\ ${ }^{2}$ Department of Macromolecular Science and Engineering, Case Western Reserve University, Cleveland, OH 7202, USA \\ ${ }^{3}$ Institut für Kunststoffe und Verbundwerkstoffe, Technical University of Hamburg-Harburg, 21073 Hamburg, Germany \\ ${ }^{4}$ CINVESTAV-Querétaro (Materials Science \& Technology), Libramiento Norponiente 2000, \\ 76230 Santiago de Querétaro, QRO, Mexico
}

Correspondence should be addressed to Luiz Antonio Ferreira Coelho, lcoelho@joinville.udesc.br

Received 3 June 2012; Accepted 3 June 2012

Copyright (c) 2012 Luiz Antonio Ferreira Coelho et al. This is an open access article distributed under the Creative Commons Attribution License, which permits unrestricted use, distribution, and reproduction in any medium, provided the original work is properly cited.

By the end of the 80s, researchers at Toyota Motor Corporation started to study the exfoliation of clay in polymeric matrices via in situ polymerization. They reinforced a polymeric matrix with small amounts of nanosized clays. In the beginning of the 90s with the Iijima's report on carbon nanotubes, an era of global nanomaterials research emerged. Initially the research focus was on the mechanical properties of the new polymer nanocomposites, but afterwards research started to be focused on the multifunctional properties of these materials. In this special issue on, the reader will find 11 selected papers briefly summarized below.

Polyaniline (Pani) and polypyrrole (Ppy) half hollow spheres with different shell thicknesses were synthesized using polystyrene (PS) as the core by Yun et al. The PS core was synthesized by emulsion polymerization. The shell thickness of the Pani and Ppy half hollow spheres can be controlled in the range of 16 to $53 \mathrm{~nm}$.

B. Akbari and R. Bagheri studied polypropylene/ montmorillonite systems prepared by melt intercalation method. The effects of compatibilizer, extruder rotor speed, and feeding rate on the properties of the nanocomposites were investigated. The results illustrate that introduction of the compatibilizer and also variations of the processing conditions affect structure and mechanical properties of the nanocomposites.

V. Pistor et al. investigated the influence of polyhedral oligomeric silsesquioxanes (POSS) nanodomains in the crystallization behavior of thermoplastic polyurethanes (TPU), and found that trisilanol isobutyl-POSS reduces crystallite sizes. Moreover, samples with higher concentrations of POSS presented two crystallization stages, forming two distinct types of crystalline structures (disks at first and then moving to spherulites). This class of polymer materials has wide potential applications, including the medical, automotive, and industrial sectors.

POSS was also used as "building blocks" for star-shaped poly(N-isopropylacrylamide) organic-inorganic hybrids, as reported by S-W. Kuo et al. These hybrids exhibited also interesting features such as photoluminescence (not observed for neat poly( $\mathrm{N}$-isopropylacrylamide) homopolymers). Apart from the interesting functional properties, the authors describe a new "variation" of atom-transfer radical polymerization process based on click-chemistry (organic azide-alkyne cyclo-addition reaction), never reported before for such systems.

Sticking into materials for optical applications, researchers reported the preparation of zinc oxide $(\mathrm{ZnO})$ nanocomposites based on poly [2-methoxy-5(2'-ethyl hexyloxy)-phenylene vinylene] (MEH-PPV) using the wellestablished spin-coating technique. In spite of the presence of $\mathrm{ZnO}$ agglomerates, the electron delocalization was not affected. The photoluminescence behavior was higher as the amount of $\mathrm{ZnO}$ in the nanocomposites increased.

Looking for antibacterial activity and interesting mechanical properties, composites of polyvinyl alcohol (PVA) reinforced with silver nanoparticles (AgnP) have been investigated by impedance spectroscopy. Combination of dielectric and dynamical-mechanical analyses gave a better 
understanding of the molecular dynamics of these materials, showing that a secondary relaxation was erroneously assigned as the glass transition of PVA and composites in former studies. Nevertheless, it has been showed that the inclusion of AgnP decreases the interfacial barrier and increases the transition probability of electron hopping across the barrier and insulator chains, providing a percolation path through the amorphous regions of the polymer matrix and thus an enhanced conductivity.

Composites have been prepared using long multiwalled carbon nanotubes (LMWCNTs, length of $2 \mathrm{~mm}$ ) and short multiwalled carbon nanotubes (SMWCNTs, length of $1 \mu \mathrm{m}$ ) by M. Zimmer et al. The use of LMWCNTs improved the electrical and mechanical properties of the composites when compared to SMWCNTs. Although the longer networks were expected to allow for more undisturbed phonon transportation, the thermal conductivity of the composites was not affected by the addition of long CNTs. These intriguing results were related to the functioning of the networks made by both the LMWCNTs and shorter MWCNTs.

The application of polystyrene/ $\mathrm{ZnO}$ nanocomposites as scattering films in lighting application has been studied by G. Nenna et al. The device efficiency was found to increase with thickness and concentration of $\mathrm{ZnO}$ nanoparticles. The resulting materials showed properties suitable for OLED lighting applications.

The paper by P. Huh and S-C. Kim presented the preparation of nanostructured composites. The work describes the formation of unique $\mathrm{ZnO}$ nanoarrays utilizing a photodynamic polymer and surface relief grating structures, demonstrating that the electrostatic layer-by-layer assembly is a simple and economical methodology.

L. Nana et al. reported how polystyrene (PS) microspheres can be first synthesized by emulsion polymerization and then used to prepare Titanium dioxide $\left(\mathrm{TiO}_{2}\right) / \mathrm{PS}$ composite microspheres by the modified sol-gel method. It was also showed that $\mathrm{Ni} / \mathrm{TiO}_{2} / \mathrm{PS}$ composite microspheres can be synthesized by the reduction and adsorption process.

Finally, the preparation of silver nanostructures from bicontinuous microemulsions was described by M. A. Pedroza-Toscano et al.

As Guest Editors for this special issue, we are pleased with diverse and intense research activities in this area. We expect the special issue will be well received by the reader as a small sample of the current research on polymer matrix nanocomposites and nanostructured materials.

\section{Acknowledgments}

The guest editors would like to acknowledge and thank authors, reviewers, and staff members of Hindawi Publishing Corporation for their contributions and support to this special issue.

Luiz Antonio Ferreira Coelho Sergio Henrique Pezzin Marcio Rodrigo Loos Luis Antonio Sanchez de Almeida Prado Alejandro Manzano Ramirez 

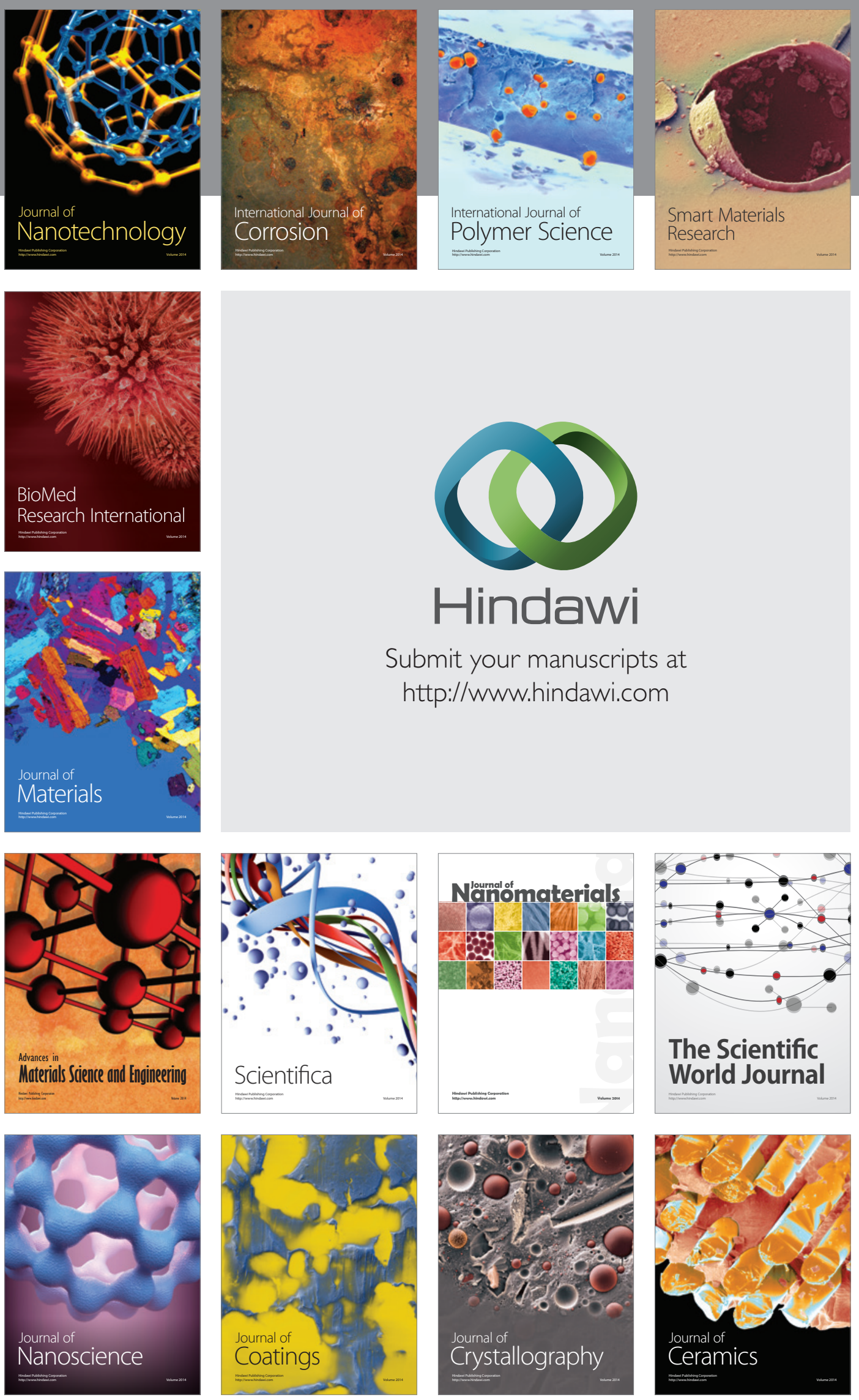

The Scientific World Journal

Submit your manuscripts at

http://www.hindawi.com

\section{World Journal}

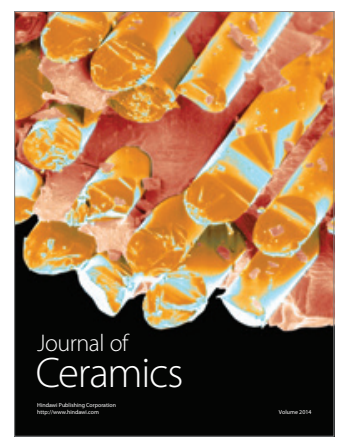

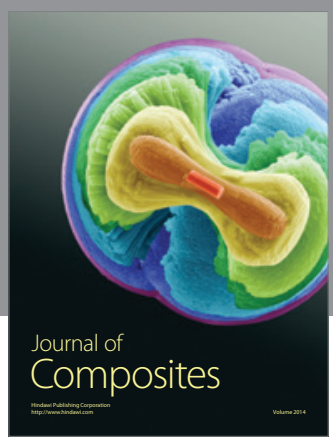
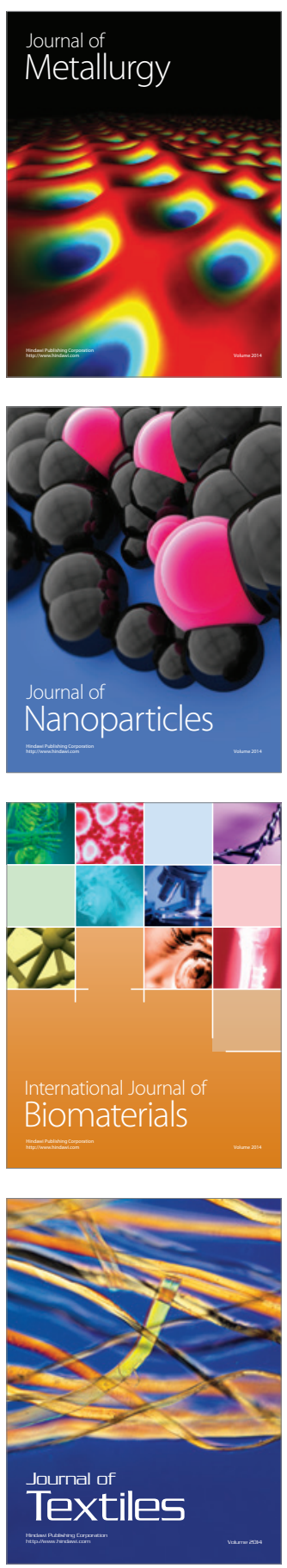